\title{
Matrix Metalloproteinase-9 Deficiency Results in Decreased Fiber Cross-Sectional Area and Alters Fiber Type Distribution in Mouse Hindlimb Skeletal Muscle
}

\author{
Ryan S. Mehan Bradley J. Greybeck Kayla Emmons William C. Byrnes \\ David L. Allen \\ University of Colorado, Boulder, Boulder, Colo., USA
}

\section{Key Words}

Myosin heavy chain - Fiber type characteristics ·

Type IV collagen

\begin{abstract}
Matrix metalloproteinases (MMPs) play a major role in the degradation of the extracellular matrix (ECM) of skeletal muscle, and the inducible gelatinase MMP-9 in particular appears to be critical for the remodeling of muscle ECM during growth and repair. Here we determined the effects of MMP9 gene inactivation on fiber type and size in the tibialis anterior (TA), gastrocnemius (GAST), and soleus (SOL) muscles in female mice. In the TA, the cross-sectional area (CSA) of the myosin heavy chain $(\mathrm{MyHC})$ llb-expressing fibers was significantly smaller in MMP-9 null mice while in the GAST, CSA of all three fast fiber types was decreased. In the SOL, MyHC type I-expressing fibers were significantly smaller in the MMP-9 null mice. The percentage of MyHC type llb-expressing fibers was significantly increased in the TA and GAST of MMP-9 null mice, while the percentage of MyHC Ild-expressing fibers significantly decreased in the GAST of MMP-9 null mice. Fiber percentages in the SOL were not significantly different between the two lines. Despite these changes in fiber
\end{abstract}

\section{KARGER}

Fax +4161306 1234

E-Mail karger@karger.ch

www.karger.com (c) 2011 S. Karger AG, Basel

Accessible online at: www.karger.com/cto size and type, in vivo hindlimb force production was not changed in MMP-9 null mice. Meanwhile, neither expression of the constitutive gelatinase MMP-2 nor immunohistochemical staining for type IV collagen was significantly altered by MMP-9 inactivation in any muscles examined. The present study demonstrates that MMP-9 inactivation results in changes in fiber size and type in adult mouse hindlimb muscles that may depend on indirect mechanisms involving reduced bone growth or nerve changes in response to MMP9 inactivation.

Copyright @ 2011 S. Karger AG, Basel

\section{Introduction}

Matrix metalloproteinases (MMPs) are a family of zinc-dependent proteases whose primary function is the degradation of the extracellular matrix (ECM) [as reviewed by Sternlicht and Werb, 2001]. In skeletal muscle, the gelatinases MMP-2 and -9 , whose primary substrate is type IV collagen, are thought to be responsible for much of the architectural remodeling that occurs within the basal lamina surrounding each muscle fiber during periods of growth or repair [Sternlicht and Werb, 2001]. 


\begin{tabular}{ll}
\hline Abbreviations used in this paper \\
\hline CSA & cross-sectional area \\
DAB & 3,3'-diaminobenzidine \\
ECM & extracellular matrix \\
F(ab) $)_{2}$ & fragment antibody 2 \\
GAPDH & glyceraldehyde-3-phosphate dehydrogenase \\
GAST & gastrocnemius muscle \\
HEFT & hindlimb exertional force test \\
IgG $(\mathrm{H}+\mathrm{L})$ & immunoglobulin G $(\mathrm{H}+\mathrm{L})$ \\
IgM $(\mathrm{H}+\mathrm{L})$ & immunoglobulin $\mathrm{M}(\mathrm{H}+\mathrm{L})$ \\
MMPs & matrix metalloproteinases \\
mRNA & messenger ribonucleic acid \\
MyHC & myosin heavy chain IIb-expressing fibers \\
OCT & optimal cutting temperature (compound) \\
PBS & phosphate-buffered saline \\
PCR & polymerase chain reaction \\
PF & peak force \\
PLT & plantaris muscle \\
RNA & ribonucleic acid \\
RT-PCR & reverse transcription-polymerase chain reaction \\
SEM & standard error of the mean \\
SOL & soleus muscle \\
TA & tibialis anterior muscle \\
WT & wild type \\
\hline
\end{tabular}

In particular, MMP-9 expression and/or activity under nonremodeling conditions are low, but are highly inducible following damage to skeletal muscle in response to exercise [Koskinen et al., 2001a, b; Rullman et al., 2007, 2009], and in response to a variety of other damaging agents or myopathic conditions [Kherif et al., 1999; Choi and Dalakas, 2000; Rucavado et al., 2002; Zimowska et al., 2008; Barnes et al., 2009]. Moreover, a wide range of cell types found within skeletal muscle can express MMP9 during postdamage repair, including endothelial cells [Kherif et al., 1998], satellite cells [Guérin and Holland, 1995], and inflammatory cells [Choi and Dalakas, 2000] as well as neurons [Kherif et al., 1998]. Given the localization patterns of MMP-9 in muscle and its temporal patterns of expression with injury, it seems likely that MMP9 plays several key roles in the remodeling of skeletal muscle ECM following injury.

What is less clear is what, if any, role MMP-9 plays in skeletal muscle during nondamage states such as normal muscle development. Given that MMP-9 typically is very minimally expressed in nonremodeling muscle, we initially hypothesized that MMP-9 gene inactivation would have no effect on noninjured skeletal muscle fiber type and size. To our surprise, muscle fiber cross-sectional area (CSA) and fiber type distribution were significantly altered in female MMP-9 null mice compared to female wild-type (WT) mice. These changes appear to occur in the absence of any glaring change in type IV collagen staining in the muscle, and moreover are not accompanied by compensatory changes in MMP-2 expression. Similarly, neither MMP-9 inactivation nor the compensatory fiber type or size changes accompanying it resulted in a change in hindlimb twitch force output in an in vivo functional test. Together, these data suggest that the inducible gelatinase MMP-9 plays a role in regulating the size of skeletal muscle fibers as well as the distribution of the various fiber types within mouse skeletal muscle, but these changes were accompanied by neither dramatic changes to the muscle basal lamina nor were they associated with a major deficit in muscle contractile function in vivo.

\section{Materials and Methods}

Antibodies and Reagents

The following primary antibodies were used for immunohistochemisty: anti-MyHC I IgG (Vector Laboratories, Burlingame, Calif., USA); anti-rabbit laminin (Sigma Chemical, St. Louis, Mo., USA), anti-MyHC IIB (SC-71 hybridoma supernatant), antiMyHC IIA (BF-F3 hybridoma supernatant), anti-MyHC IID (6H1 hybridoma supernatant) were all generously provided by Dr. Leslie Leinwand (University of Colorado, Boulder, Boulder, Colo., USA). Rabbit polyclonal antibody to collagen IV IgG was from AbCam (Cambridge, Mass., USA). Secondary antibodies used for immunohistochemistry were peroxidase-conjugated goat antirabbit IgG $(\mathrm{H}+\mathrm{L})$ (Vector Laboratories, Burlingame, Calif., USA), peroxidase-conjugated goat anti-mouse IgG $(\mathrm{H}+\mathrm{L})$, and peroxidase-conjugated Affini-Pure $\mathrm{F}(\mathrm{ab})_{2}$ goat anti-mouse $\operatorname{IgM}(\mu$ chain specific) (Jackson ImmunoResearch, West Grove, Pa., USA). The DAB substrate kit for peroxidase was from Vector Laboratories.

Mice

All experimental procedures involving mice were approved by the Institutional Animal Care and Use Committee of the University of Colorado, Boulder, and complied with the guidelines of the American Physiological Society on the use of laboratory animals. Female WT C57/black6j and MMP-9 null mice (for the muscle mass analysis $\mathrm{n}=5$ for WT and $\mathrm{n}=7$ for MMP- 9 null; for the fiber size and type analysis $n=3$ or 4 animals per genotype) were obtained from our breeding colony in the Department of Integrative Physiology at the University of Colorado, Boulder. Female mice were used because male MMP-9 null mice were used for other studies involving muscle injury, which will be reported elsewhere. MMP-9 null mice were originally obtained from Dr. Robert Senior at Washington University School of Medicine in St. Louis, Mo., USA and were bred in our mouse colony at the University of Colorado, Boulder. These mice are viable and fertile and have no gross defects in skeletal muscle [Vu et al., 1998]. All mice were between 2.5 and 3.5 months in age. 


\section{Tissue Collection}

Mice were anesthetized with isoflurane (VetOne, Meridian, Idaho, USA) and sacrificed by cervical dislocation.

Tibialis anterior (TA), gastrocnemius (GAST), and soleus (SOL) muscles were removed bilaterally, weighed, and mounted in Tissue-Tek Optimal Cutting Temperature (OCT) Compound (Sakura Finetek USA, Inc., Torrance, Calif., USA) on cork blocks, and snap frozen in isopentane chilled in liquid nitrogen, and stored at $-80^{\circ} \mathrm{C}$. Care was taken to make sure muscles were frozen at or near resting length for both WT and MMP-9 null muscles; we observed no systematic difference in freezing length between WT and MMP-9 null muscles (data not shown).

\section{Histology}

Frozen serial transverse cross-sections $(10 \mu \mathrm{m})$ from the midbelly of each muscle were cut at $-20^{\circ} \mathrm{C}$ on a Leica CM1900 cryostat as previously described [Allen et al., 2001; Harrison et al., 2002, 2003, 2010b]. Briefly, a chunk was cut from the muscle midbelly using a sharp new razor blade and mounted vertically using OCT. Care was taken to cut and mount all muscle chunks vertically so as to avoid large or systematic differences in sectioning angle. All sections were placed on gelatin-coated slides and stored at $-20^{\circ} \mathrm{C}$ until immunohistochemical staining.

For anti-MyHC I, IIa, IIb, and IId immunohistochemical analysis, frozen serial muscle sections were thawed and air-dried at room temperature. Sections were incubated in blocking solution ( $3 \%$ bovine serum albumin, $0.05 \%$ Tween -20 , and $0.2 \%$ gelatin in phosphate-buffered saline, PBS) at room temperature for $1 \mathrm{~h}$. Samples were then incubated with the appropriate primary antibody solution at room temperature for $2 \mathrm{~h}$. The primary antibody solutions were anti-MyHC I (1:40 dilution in blocking solution), anti-MyHC IIa (1:3 in blocking solution), anti-MyHC IIb (1:4 dilution in blocking solution), and anti-MyHC IId (1:20). Anti-laminin antibody (1:40 dilution in blocking solution) was added to the primary antibody solution to help visualize fibers. Following incubation with primary antibodies, slides were washed three times for 5 min with PBS, and then incubated with secondary antibodies for $1 \mathrm{~h}$ at room temperature. The secondary antibody solutions were either peroxidase-conjugated goat anti-mouse $\operatorname{IgG}(\mathrm{H}+\mathrm{L})$ at 1:50 dilution in blocking solution (for $\mathrm{MyHC}$ I and $\mathrm{MyHC} \mathrm{IIa),} \mathrm{or}$ peroxidase-conjugated Affini-Pure $\mathrm{F}(\mathrm{ab})_{2}$ goat anti-mouse IgM ( $\mu$-chain specific) at 1:50 dilution in blocking solution (MyHC IIb) and peroxidase-conjugated goat anti-rabbit $\operatorname{IgG}(\mathrm{H}+\mathrm{L})$ at 1:50 dilution in blocking solution (for laminin). After three 5-min washes in PBS, the slides were developed with 3,3'-diaminobenzidine (DAB) substrate (prepared as directed by the manufacturer with addition of nickel solution) for $5 \mathrm{~min}$ at room temperature. Sections of WT and MMP-9 null muscle were always stained together in pairs so as to avoid large or systematic differences in staining intensity between genotypes.

Representative micrographs were taken using the Motic USB 2.0 camera (Moticam $20002.0 \mathrm{M}$ pixel USB 2.0P, and Motic Images Plus 2.0 software, Motic China Group Co.). Images of 5-8 fields were captured using a $40 \times$ lens objective for 2-3 sections of each muscle type $(n=75-385$ total fibers were analyzed for each type of muscle and averaged for each mouse). In sections used for muscle fiber type analysis, each muscle fiber in a given field was assigned as positively or negatively stained based on whether it stained above background for the particular $\mathrm{MyHC}$ isoform antibody. Fiber type CSA analysis was performed using the 'measure' function of the Motic Images Plus 2.0 software. WT and MMP-9 null sections were always analyzed together in pairs so as to avoid major discrepancies in interpretations of fiber staining between genotypes.

Threshold analysis was used to determine collagen-staining quantity. Threshold analysis was performed by first air-drying frozen serial muscle sections of TA, GAST and SOL at room temperature for $10 \mathrm{~min}$. Sections were incubated in blocking solution at room temperature for $1 \mathrm{~h}$, followed by a 2-hour incubation at room temperature with rabbit polyclonal antibody to collagen IV (diluted 1:50 in blocking solution). Sections were washed three times for 5 min with PBS, and then incubated for $1 \mathrm{~h}$ at room temperature with secondary antibody solution [peroxidase-conjugated goat anti-rabbit IgG $(\mathrm{H}+\mathrm{L})$ diluted 1:50]. After three 5-min washes in PBS, the slides were developed with DAB substrate (prepared as described above).

Images of 6 fields per section were captured using a $40 \times$ lens objective for 2 sections of each muscle type. Images were then opened in ImageJ64 (National Institutes of Health, USA) and converted into 8-bit grayscale photos. Threshold was adjusted for artifacts and ECM by adjusting the histogram of gray values to levels where red appeared only in the non-cell areas of the image. Background was converted to dark, and the threshold was adjusted until only the ECM was not selected. The area fraction of the non-ECM portion of the sample was then determined and the percentage of collagen staining in the photo was determined by subtracting the sum of the artifact area fraction and cell area fraction from $100 \%$. This allowed us to correct for differences in fiber size between the two genotypes (see below) by expressing the total ECM-staining area relative to fiber area.

\section{Reverse Transcription Polymerase Chain Reaction}

Levels of MMP-2 mRNA were evaluated using the techniques recently used by us to quantify myostatin pre-mRNA [Allen et al., in press]. Total RNA was isolated from GAST/plantaris (PLT) and SOL muscles ( $\mathrm{n}=5 \mathrm{WT}$ and $\mathrm{n}=5 \mathrm{MMP}-9$ null) with Trizol reagent (Invitrogen) using standard techniques [Allen et al., 2010c]. RNA concentration was determined spectrophotometrically at A260 and brought to a concentration of $100 \mathrm{ng} / \mu \mathrm{l} .250 \mathrm{ng}$ of RNA was then used for each reaction in a $25-\mu l$ reaction volume. A reverse transcription polymerase chain reaction (RT-PCR) was carried out using the One-Step RT-PCR kit from Qiagen according to the manufacturer's instructions. The primer sequences for MMP-2 were as follows: 5'-TGTGTCTTCCCCTTCACTTT-3') and 5'-GATCTGAGCGATGCCATCAA-3'; as a control, primers for GAPDH the following primers were used (forward: $5^{\prime}$-ATCACCATCTTCCAGGAGCGA-3'; reverse: 5'-AGTCTTCTGGGTGGCAGTGAT- $\left.3^{\prime}\right)$. The reverse transcription reaction was carried out for $30 \mathrm{~min}$ at $50^{\circ} \mathrm{C}$; this was followed by the initial PCR activation step of $95^{\circ} \mathrm{C}$ for $30 \mathrm{~min}$. Thirty-five cycles of denaturation $\left(1 \mathrm{~min}\right.$ at $\left.94^{\circ} \mathrm{C}\right)$, annealing $\left(45 \mathrm{~s}\right.$ at $\left.55^{\circ} \mathrm{C}\right)$ and extension $(45 \mathrm{~s}$ at $72^{\circ} \mathrm{C}$ ) were followed by $10 \mathrm{~min}$ of final extension at $72^{\circ} \mathrm{C}$. A 5 - $\mu$ l volume of $6 \times$ loading buffer was added to each sample and the entire reaction was run on a $1.5 \%$ agarose gel containing 10 $\mu \mathrm{g} / \mathrm{ml}$ ethidium bromide, and visualized and photographed using a gel documentation system. Gel images were scanned and the bands for MMP-2 mRNA and GAPDH were quantified by densitometry using NIH Image software. MMP-2 band intensities were normalized to those of the corresponding GAPDH band intensity for each sample. 
Hindlimb Exertional Force Test

Hindlimb exertional force test (HEFT) was carried out as previously described [Cannon et al., 2005; Hanson et al., 2010]. This test has been previously used to evaluate functional changes accompanying muscle atrophy in response to decreased loading [Cannon et al., 2005; Hanson et al., 2010], and produces similar results as in vitro muscle physiological testing [Hanson et al., 2010]. Briefly, WT and MMP-9 null mice ( $\mathrm{n}=6$ each) were first placed in a prefabricated polycarbonate cylindrical tube which had a slot measuring $6 \mathrm{~mm}$ wide at the top of the tube along the longitudinal axis and continuing across the back side of the tube to allow the mouse to be placed in the device by the tail. Once in the tube, a wood dowel plus swivel apparatus was secured to the tail of the mouse approximately $6.5 \mathrm{~mm}$ from the base end of the tail with biocompatible adhesive tape. A hook on the other end of the swivel apparatus was attached to a horizontally mounted 2-lb thin beam force transducer (TBS-2, Transducer Techniques, Temecula, Calif., USA). The tube was then moved onto the HEFT test stand such that there was little or no slack in the tail. Inside the tube, the mouse forelimbs rested on the smooth surface of the tube and the hindlimbs rested on a silicone mat placed at the tail end of the tube to provide a consistent gripping surface. Conductive gel was applied to the tail and two metal clamps were placed on the tail approximately $25 \mathrm{~mm}$ apart for a stimulating shock. The mouse was allowed to acclimatize to the tube environment for 3 min with a silicone mat draped over the tube to decrease room light and animal anxiety. After the $3 \mathrm{~min}$, the HEFT consisting of two tests began with force transducer signal sampling at 1,000 samples per second. The forward thrust of the mouse was reproducibly invoked by applying a shock $(1.5 \mathrm{~mA})$ for $300 \mathrm{~ms}$. Data collection continued for $3 \mathrm{~s}$ starting $200 \mathrm{~ms}$ prior to the onset of the stimulating shock. A second HEFT was then performed separated from the first by $2 \mathrm{~min}$, and the maximum peak force (PF) from both tests was taken. During each test, the force transducer signal was measured via a custom-designed LabviewTM v7.1 Virtual Instrument (VI; National Instruments Corp., Austin, Tex., USA) as a function of time. A computer algorithm written in MATLABTM R2010 was used for postprocessing to determine the PF.

\section{Statistics}

All values are reported as means and standard errors of the means (SEM). For fiber size and type analysis, 75-385 fibers from each hindlimb muscle were analyzed and then averaged for each of the 3 or 4 mice per genotype. Fiber size, fiber percentage, collagen staining, MMP-2 mRNA levels, and HEFT force values were compared across different mouse strains using an independentsamples t test. A level of 0.05 was used to indicate significance.

\section{Results}

\section{Body Mass and Tissue Mass}

The average body mass of the WT mice was $23.6 \mathrm{~g} \pm$ $2.3 \mathrm{~g}$, while the average body mass of the MMP-9 null mice was $20.0 \mathrm{~g} \pm 0.5 \mathrm{~g}$; this reduction in body mass is statistically significant. Absolute muscle mass was significantly decreased for the GAST but was not significantly different for the TA or SOL of MMP-9 null mice (fig. 1a). When normalized to body mass, there was no significant difference between MMP-9 null and WT mice in mass of any of the muscles analyzed (fig. 1b).

\section{Muscle Fiber CSA}

As seen in figure 2a, the CSA of MyHC IIb-expressing fibers was significantly smaller ( $16.5 \%$ smaller) in the TA of MMP-9 null mice than in WT mice. There was no significant difference in the CSA of MyHC type I, IIa, or IId-expressing fibers in TA muscles between the two lines. In GAST, the CSAs of MyHC type IIa (33.3\% smaller)-, IId (29.4\% smaller)- and IIb (34.6\% smaller)-expressing fibers were all significantly smaller in MMP-9 null mice than in WT mice (fig. 2b). The CSA of type I MyHCexpressing fibers found in MMP-9 null GAST was not significantly different from those of the WT mice.

Analysis of the SOL muscle also revealed significant differences in fiber CSA between the two lines. The CSA of MyHC I-expressing fibers (11.3\% smaller) was modestly but significantly smaller in the SOL of MMP-9 null mice compared to WT mice (fig. 2c). The MyHC type IIa- and IIb-expressing fibers of MMP-9 null SOL were not significantly different from those in WT mice; however, the fact that the difference in MyHC IIb-expressing fibers was not statistically significant may have been due in part to the small number of MyHC type IIb-expressing fibers in the SOL in either line (8 fibers in WT and 25 in MMP-9 null mice). No MyHC type IId-expressing fibers were observed in the SOL of WT mice and only 6 were found in MMP-9 null mice, which precluded comparison of the CSA of IIdexpressing fibers between genotypes in this muscle.

Figure 3 shows representative panels of $\mathrm{MyHC}$ immunohistochemical staining for the GAST from WT and MMP-9 null mice.

\section{Muscle Fiber Type Distribution}

In the TA of both WT and MMP-9 null mice, MyHC type IIb-expressing fibers accounted for a large portion of the total fibers, and in MMP-9 null mice (about 62\%) this distribution increased significantly compared to WT mice (about 50\%) (fig. 4a). The remaining fibers that comprise the TA in both lines were primarily MyHC type IId-expressing (54\% in WT and 46\% in MMP-9 null mice) or MyHC type IIa-expressing (about $34 \%$ in WT and about $40 \%$ in MMP-9 null mice), and the percentage of these fiber types did not differ statistically between the two lines. Note that due to coexpression of $\mathrm{MyHC}$ isoforms, particularly IId with the other fast isoforms, the percentages add up to more than $100 \%$, which also did 

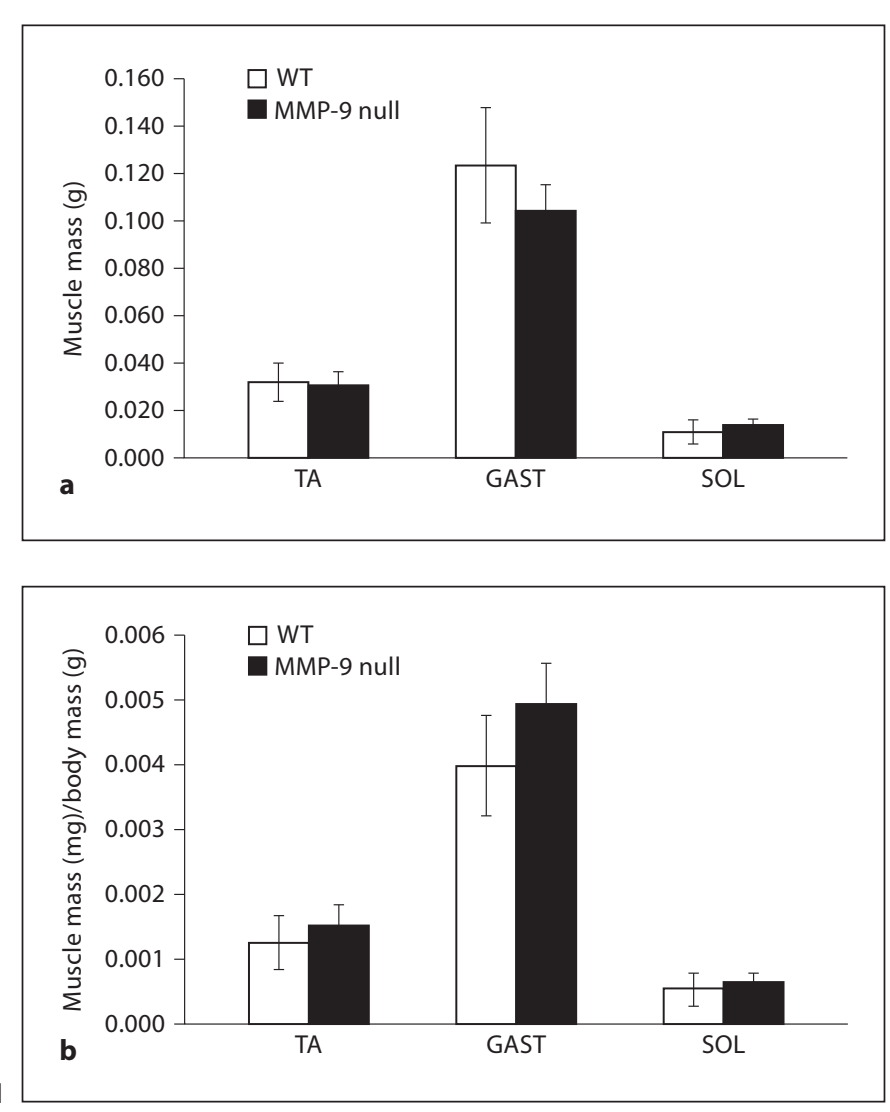

Fig. 1. Absolute and relative muscle mass for WT and MMP-9 null mice. a Absolute muscle mass in grams for TA, GAST and SOL. b Relative muscle mass (normalized to body weight) for TA, GAST and SOL. When normalized to body mass there are no differences in TA, GAST or SOL muscle mass of MMP-9 null mice compared to WT mice. Values are means \pm SEM for $5 \mathrm{WT}$ mice and 7 MMP-9 null mice.

Fig. 2. Mean muscle fiber CSA of WT and MMP-9 null mice for the TA (a), GAST (b) and SOL (c) muscles. Values are means \pm SEM for 3 or 4 mice per genotype, with the exception of WT TA MyHC I (3 fibers), MMP-9 null TA MyHC I (7 fibers), WT SOL MyHC IId (0 fibers), MMP-9 null SOL MyHC IId (6 fibers), WT SOL MyHC IIb (8 fibers) and MMP-9 null SOL MyHC IIb (25 fibers). ${ }^{*} \mathrm{p}<0.05$, significantly different from WT.

not differ between the two lines in any of the hindlimb muscles examined. Less than $3 \%$ of the total fibers found in TA muscle in either line were MyHC type I-expressing fibers, and these did not differ between the two lines.

As in the TA, MyHC IIb-expressing fibers represented the majority (about 56\% in WT and about 79\% in MMP9 null) fiber type in GAST (fig. 4b), and as in TA, the increase in the percentage of MyHC type IIb-expressing fibers in the GAST of MMP-9 null mice was statistically significant compared to WT mice (fig. 4b). There were
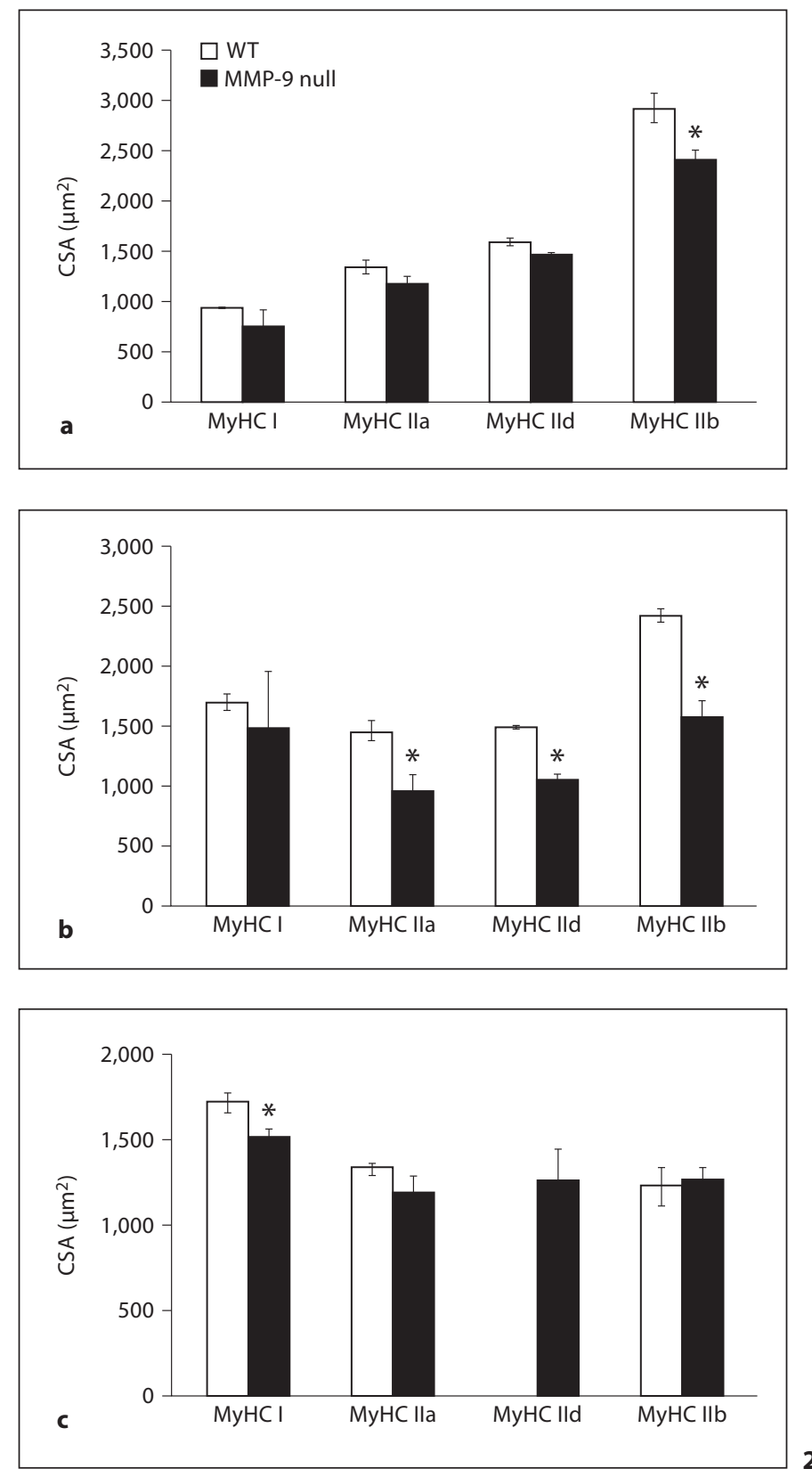

also significantly fewer MyHC type IId-expressing fibers in the GAST of MMP-9 null mice (16\%) compared to WT mice (33\%) (fig. 4b). In both lines, between $18-20 \%$ of the GAST fibers expressed MyHC type IIa, while MyHC Iexpressing fibers accounted for less than $14 \%$ of the total fibers (fig. 4b), and these did not differ statistically between the two lines.

In SOL of both lines of mice, MyHC type I- and IIaexpressing fibers account for roughly equal proportions of the muscle fibers, while less than $4 \%$ of the SOL fibers 


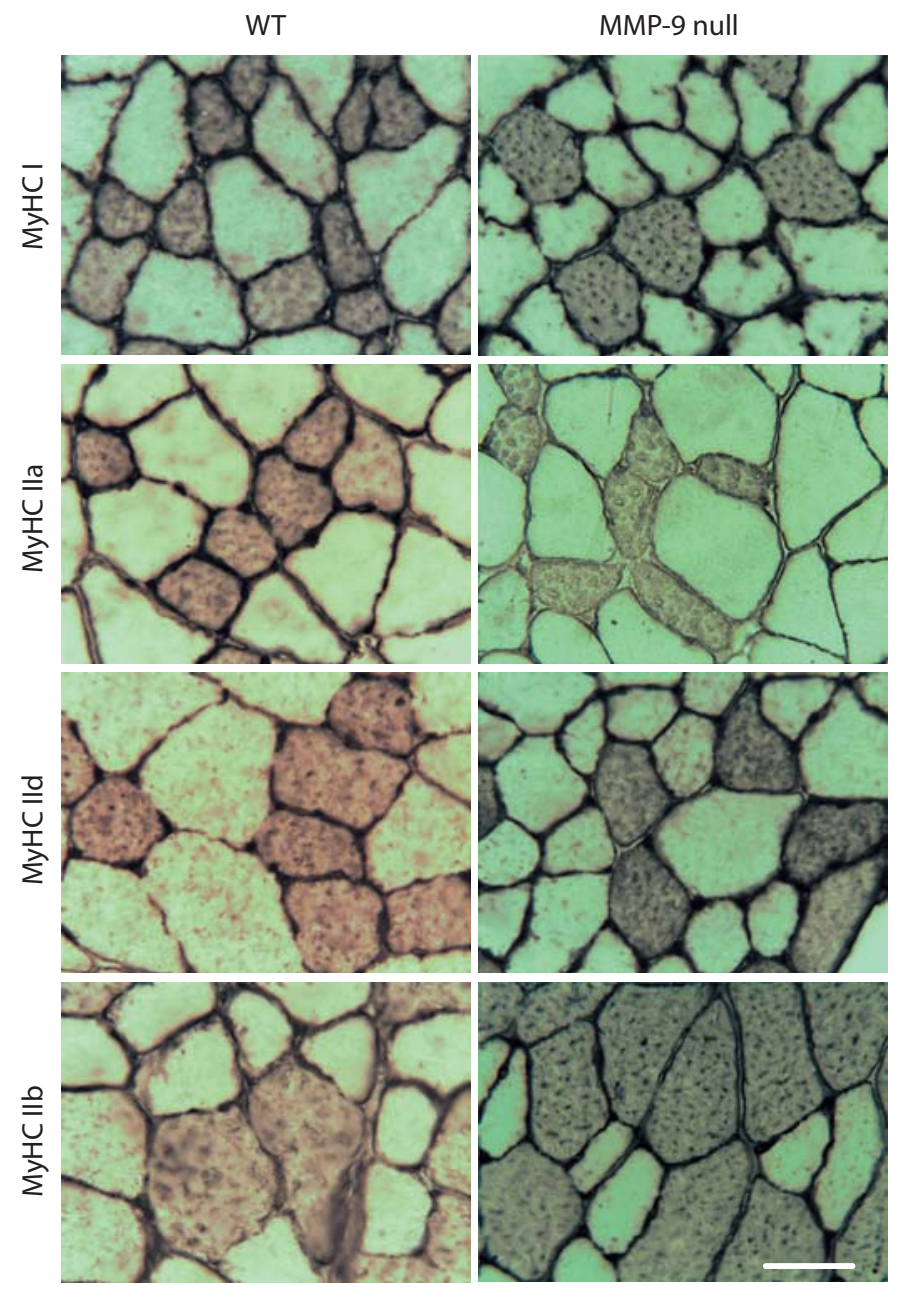

Fig. 3. Immunohistochemical staining of the GAST muscle from WT (left column) and MMP-9 null (right column) mice. The two top panels show staining for MyHC I and laminin; the second row of panels from the top show staining for $\mathrm{MyHC}$ IIa and laminin; the third row of panels show staining for MyHC IId and laminin; the bottom two panels show staining for MyHC IIb. Scale bar = $50 \mu \mathrm{m}$.

were type IIb or type IId for either line (fig. 4c). The two lines of mice had no significant differences in distribution of MyHC I-, IIa-, IId-, or IIb-expressing fibers in the SOL (fig. 4c).

\section{Type IV Collagen Staining and MMP-2 Expression}

Figure 5a shows representative sections from WT and MMP-9 null mice immunohistochemically stained for type IV collagen. Qualitatively, no major differences are obvious (fig. 5a). Threshold analysis revealed that of the three muscles studied none displayed a significant differ-
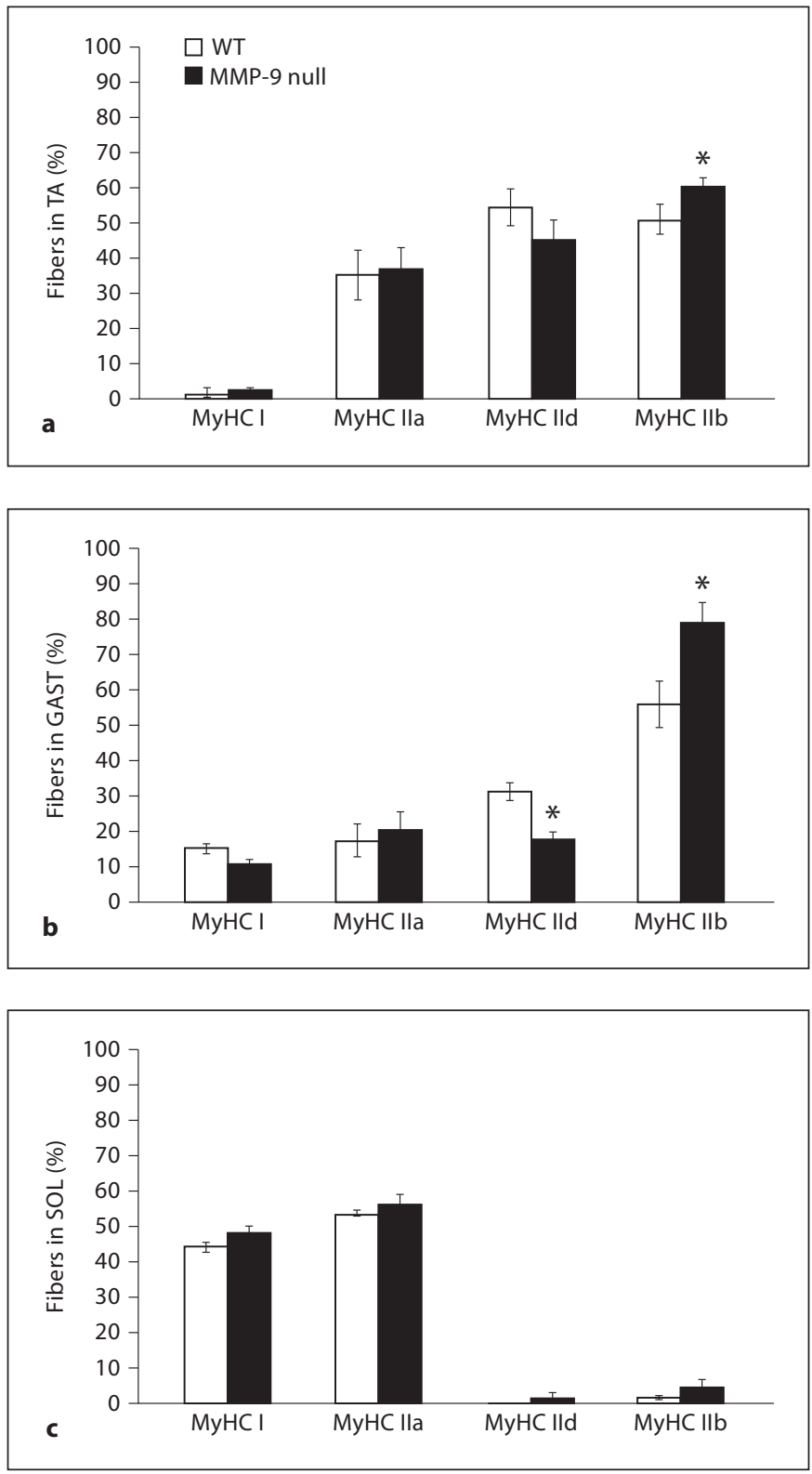

Fig. 4. Fiber type percentages of WT and MMP-9 null mice TA (a), GAST (b) and SOL (c). Values are means \pm SEM for 40-385 fibers from 3 or 4 muscles per genotype. ${ }^{*} \mathrm{p}<0.05$, significantly different from WT.

ence in the relative percentage of type IV collagen staining between C57 WT and MMP-9 null mice. In both lines of mice, the muscle with the largest relative percentage of type IV collagen staining was the SOL. In WT SOL, the area of collagen staining was $21.6 \pm 1.3 \%$, compared to $19.4 \pm 3.9 \%$ in the MMP-9 null mice (fig. 5b). WT TA 


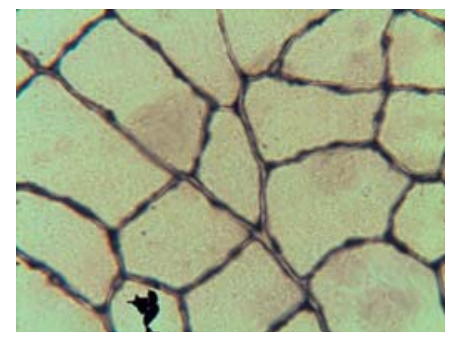

a

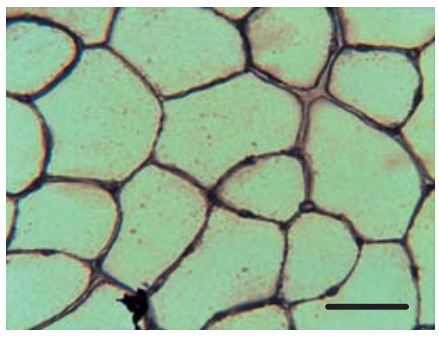

MMP-9 null
Fig. 5. Collagen staining analysis. a Representative immunohistochemical staining for type IV collagen on cross-sections of WT and MM9-9 null TA stained for type IV collagen. Scale bar $=50 \mu \mathrm{m}$.

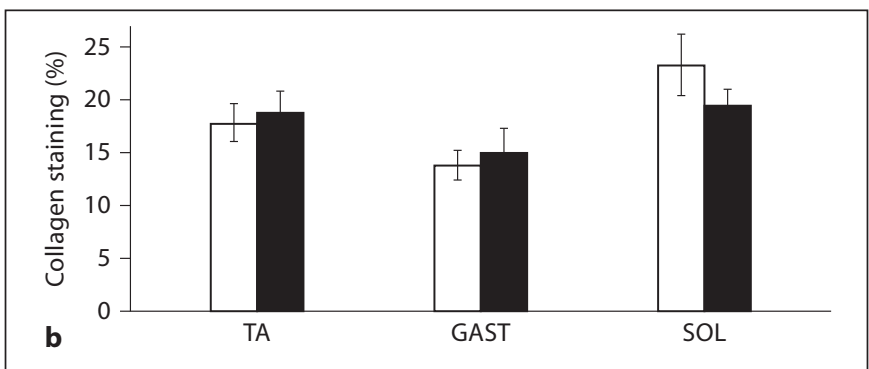

b Type IV collagen staining quantification by threshold image analysis in the TA, GAST and SOL muscle of WT and MMP-9 null mice. Values are means \pm SEM for 3 or 4 animals per condition.
Fig. 6. RT-PCR analysis of MMP-2 mRNA expression in WT and MMP-9 null mice. a Agarose gel showing MMP-2 and GAPDH for WT (left) and MMP-9 null (right) GAST/PLT. b Bar graph quantifying the results for 5 WT and 5 MMP-9 null GAST/PLT muscles. MMP-2 is normalized to GAPDH; bars represent mean \pm SEM. c Agarose gel showing MMP-2 and GAPDH for WT (left) and MMP-9 null (right) SOL. d Bar graph quantifying the results for $6 \mathrm{WT}$ and 6 MMP-9 null SOL muscles. MMP-2 is normalized to GAPDH; bars represent mean \pm SEM.

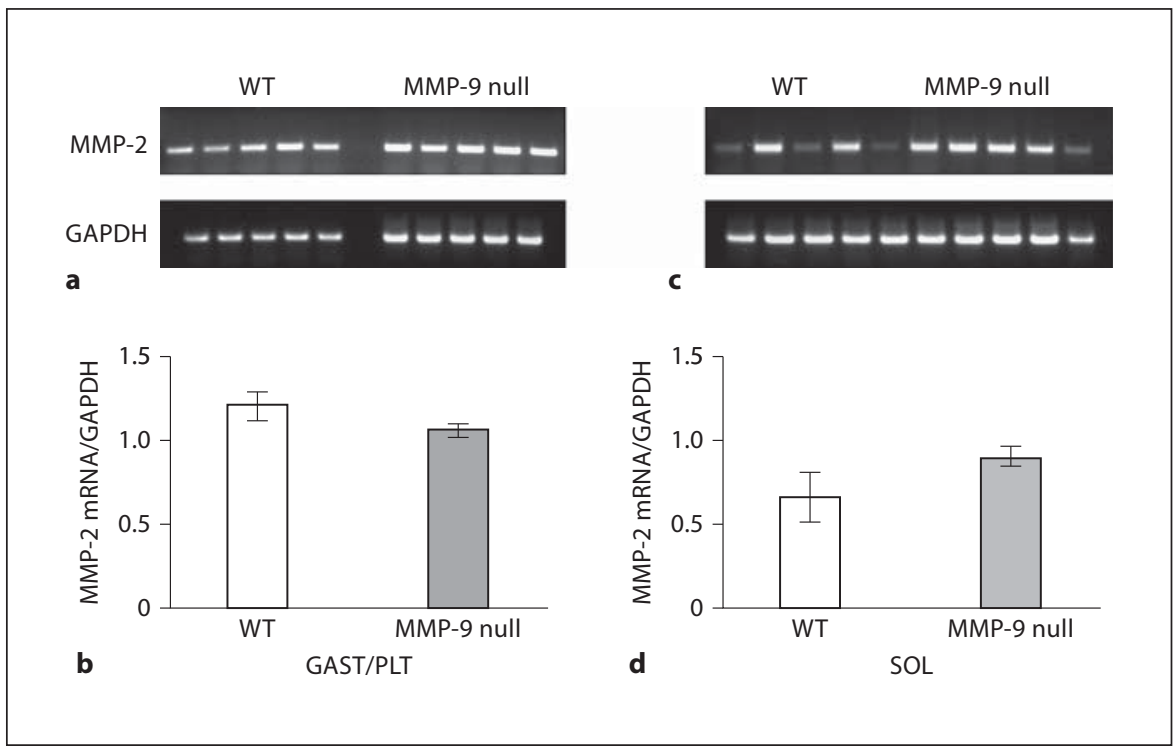

contained $17.7 \pm 0.85 \%$ type IV collagen area staining, while the TA in MMP-9 null mice was $17.9 \pm 0.94 \%$ (fig. 5b). In both genotypes, the GAST was found to display the lowest relative percentage of type IV collagen staining compared to the other two hindlimb muscles, though the difference was not significant. In this muscle, WT mice had $13.7 \pm 0.80 \%$ type IV collagen staining, while MMP-9 null GAST had $15.5 \% \pm 1.2$ type IV collagen staining.

We then examined the effects of MMP-9 inactivation on MMP-2 mRNA expression in both a fast (GAST/PLT) and slow (SOL) hindlimb muscle/muscle group. Inactivation of MMP-9 had no effect on MMP-2 mRNA levels in either GAST/PLT or SOL compared to WT (fig. 6a-d).

\section{Functional Studies with HEFT}

We also used an in situ hindlimb contraction test, known as the HEFT, to ascertain the effects of both MMP-9 inactivation and its consequences on hindlimb force production. As can be seen in figure 7 , maximal force production was not significantly different between WT and MMP-9 null mice.

\section{Discussion}

In the present study, we investigated the effects of the absence of MMP-9, an inducible regulator of ECM remodeling [Sternlicht and Werb, 2001], on skeletal mus- 


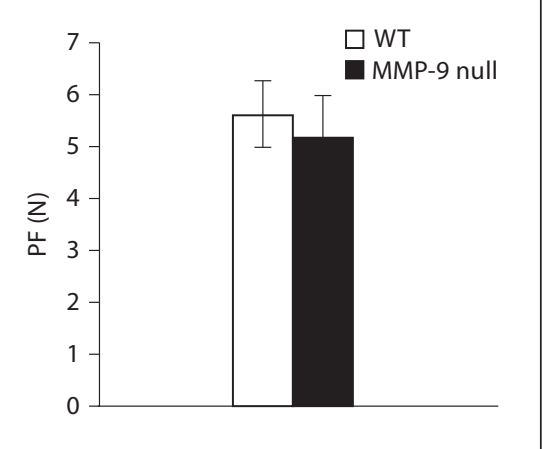

Fig. 7. Hindlimb force production for WT and MMP-9 null mice. Bar graph showing the average PF in the HEFT in vivo twitch test for WT and MMP-9 null mice. Bars represent mean \pm SEM for 6 WT and 6 MMP-9 MMP-9 null mice.

cle fiber size and type distribution, as well as on type IV collagen staining area in mice. We demonstrate that both muscle fiber CSA of specific fiber types and fiber type percentages were altered in several hindlimb muscles of MMP-9 null mice compared to WT mice. Together, these data suggest that the absence of MMP-9 results in decreased muscle fiber size which could occur due to attenuation in either prenatal or postnatal muscle growth.

MMP-9 Inactivation Decreases Both Muscle Mass and Overall Body Mass

The MMP-9 null mice were found to have an about 3.6-gram average body mass reduction when compared to the WT mice. This reduction in body mass may, in part, be due to a reduction of muscle mass in the MMP-9 null mice. Absolute muscle mass was only significantly different for the GAST muscle due to variability (fig. 1a), but muscle mass normalized to body mass was unchanged. Since whole body mass was significantly decreased, the fact that muscle mass normalized to body mass does not decrease in MMP-9 null mice suggests that the muscles of these mice decreased proportionally to body mass, which in turn suggests that these muscles underwent decreased growth rather than true atrophy in response to MMP-9 inactivation. Muscles other than those presently studied may also have been affected by MMP-9 inactivation and may therefore have contributed to the differences in mean body mass between the two lines of mice. In addition to skeletal muscle, the absence of MMP-9 might also have affected the mass of other lean and fat tissues. Recent work has uncovered a link between adiposity levels and MMP-9 levels in children [Kaditis et al., 2010]. Although it has not yet been determined whether MMP-9 plays a causal role in regulating fat mass, it is possible that the absence of this enzyme may have affected fat mass through a similar mechanism.

\section{Absence of MMP-9 Results in Changes in Fiber Size and Type in Hindlimb Muscles}

In addition, the absence of MMP-9 significantly decreased the CSA of different types of skeletal muscle fibers expressed in the TA, GAST and SOL. This effect was most dramatic in MyHC type IIb-expressing fibers in fast muscles (TA and GAST) of the lower leg. However, the reduction in fiber size brought about by the absence of MMP-9 was not limited to the fast muscles; in the SOL, CSA of MyHC type I-expressing fibers was also significantly smaller in MMP-9 null mice. Thus, the effects of MMP-9 inactivation on fiber CSA do not appear to be fiber or muscle specific, which suggests that the decreases in fiber size in MMP-9 null mice are not a consequence of decreased muscle activation, which tends to affect slow-twitch muscles and fibers preferentially [Roy et al., 1991], but are instead a generalized response of all hindlimb muscles regardless of type.

In addition to changes in fiber CSA, MMP-9 null mice exhibited alterations in fiber type distribution. In MMP9 null mice the fiber types that had the largest decrease in CSA in the fast-twitch hindlimb muscles studied were also observed to display an increase in fiber type percentage distribution. Specifically, the percentage of $\mathrm{MyHC}$ IIb-expressing fibers was greater in both the TA and GAST of MMP-9 null mice. The increase in MyHC type IIb fiber percentage in these muscles may have been, at least partly, a physiological adaptation designed to offset the decrease in fiber CSA so as to maintain the total functional CSA or fiber mass [Armstrong and Phelps, 1984] of this fiber type, which is the average fiber CSA multiplied by the fiber percentage. Moreover, shifts toward more faster-twitch fiber types with atrophy may offset some of the loss in force production caused by decreases in fiber CSA by increasing the rate of force production, which in turn attenuates the decrease in power output, since power output is correlated with the amount of fasttwitch fibers within a muscle [Fitts et al., 1991]. Interestingly, however, the MyHC type IId-expressing fibers of the MMP-9 null GAST had a smaller mean CSA and also had a decreased percentage when compared to WT mice. Combined with the loss of type IId CSA, it would seem that in the GAST of MMP-9 null mice the total functional CSA of MyHC type IId-expressing fibers is decreased. 
MMP-9 Inactivation and Hindlimb Force Production

Interestingly, despite these changes in muscle fiber CSA and type, in vivo force production as assessed by the HEFT was not significantly different between WT and MMP-9 null mice. This test has been used previously to quantify the decrements in force output accompanying the muscle atrophy and fiber type shifts occurring in mice in response to hindlimb unloading [Cannon et al., 2005; Hanson et al., 2010]. It is not clear why we observed no significant changes in HEFT in the present study, but it may be that the changes in muscle and fiber size reported here were not of sufficient magnitude to be detected by the HEFT. Alternatively, it may be that muscles of MMP-9 mice undergo adaptations that compensate for the decreases in muscle fiber size. Nonetheless, these results suggest that neither MMP-9 inactivation nor its downstream consequences adversely affected in vivo twitch force generation.

\section{MMP-9 Inactivation and Muscle Variables}

The primary biological substrate of the MMP-9 enzyme is type IV collagen. Inactivation of the gene for MMP-9 could be predicted to result in alterations of type IV collagen composition within muscle tissue, and therefore affect the establishment and/or maintenance of muscle fiber size. However, no significant differences were observed between the two lines of mice for percentage of staining of collagen IV in muscle sections in the TA, GAST or SOL, though it should be pointed out that this assay does not eliminate the possibility that changes in collagen density may exist between the two genotypes. This nevertheless suggests that the changes observed in fiber type and size in the present study in response to MMP-9 inactivation were not due to gross changes in adult muscle fiber ECM quantity.

In addition, we examined the effects of MMP-9 inactivation on the expression of the constitutive gelatinase MMP-2. There is some evidence that both basal [Ovechkin et al., 2005] and induced [Ducharme et al., 2000; Tang et al., 2004; Ikonomidis et al., 2005] MMP-2 expression and/or activity are increased in other tissues of MMP-9 null mice, and an increase in MMP-2 activity could conceivably cause changes in ECM degradation and/or growth factor activation resulting in changes in muscle fiber growth or type. However, we observed no significant difference in MMP-2 mRNA levels in either the fast-twitch (GAST/PLT) or slow-twitch (SOL) muscles of the hindlimb, suggesting that MMP-2 expression was not elevated as a consequence of MMP-9 inactivation, and thus probably did not contribute to the differ- ences in fiber type and size observed in the present study.

Thus, at present, the mechanisms responsible for the effects of MMP-9 inactivation on muscle fiber type and size are not clear but do not appear to involve changes in either collagen staining or compensatory MMP-2 expression. At least three other possible mechanisms for the effects of MMP-9 on muscle fiber type and size can be envisioned. First, it may be that MMP-9 is expressed in skeletal muscle during some key period of muscle fiber formation during pre- or postnatal development, and inactivation of the MMP-9 gene during this key period results in changes in muscle fiber type and size that remain during the postnatal state. During development, remodeling of the ECM is a potent contributor, both mechanically and biochemically, to the process of tissue morphogenesis [Ingber, 2006], and MMPs play many key roles during development [Vu and Werb, 2000]. MMP-9, in particular, is expressed in myoblasts in vitro [Allen et al., 2003] and appears to play a crucial role in the migration of myoblasts [Lewis et al., 2000] and also modulates myotube size in vitro [Lluri et al., 2008].

Alternatively, MMP-9 inactivation may influence muscle fiber size and type indirectly through effects on the motor nerve and/or on the amount or pattern of neuromuscular activation. MMP-9 protein is expressed by motor neurons and Schwann cells and is enriched in the neuromuscular junction of uninjured muscle [Kherif et al., 1998] and thus MMP-9 gene inactivation may influence neural activation of skeletal muscle contraction and/or activation of trophic compounds released by the nerve. However, several lines of evidence argue against a major defect in neuromuscular transmission or muscle activity levels being responsible for the changes observed here. First, as mentioned above, the pattern of CSA decrease does not support an activity-dependent form of atrophy or reduced growth. Second, with respect to gross patterns of motor activity, we did not observe any differences in either the pattern or volume of cage locomotion or in the ability to engage in a $3 \times 30 \mathrm{~min}$ treadmill run between $\mathrm{WT}$ and MMP-9 null mice [unpublished observations]. Third, the data presented in figure 7 demonstrate that maximal in situ twitch force production in the HEFT was not significantly different between WT and MMP-9 null mice, again consistent with the interpretation that major defects in excitation-contraction do not exist in the MMP-9 mice. Thus, at present, the absence of MMP-9 does not appear to produce decrements in twitch force development in vivo or in gross motor behavior that would tend to explain the effects of MMP-9 inactivation on muscle fiber type and size. 
Finally, there is some evidence that points to an influence of MMP-9 inactivation on bone growth during development, and this could have indirectly affected muscle fiber growth through decreased postnatal bone growth. Vu et al. [1998] reported that MMP-9 is involved in endochondral bone formation, and that neonatal and young mice lacking MMP-9 had reductions in bone ossification, resulting in delayed bone development and reduced postnatal body growth. Therefore, such changes in bone growth could have had a dramatic indirect impact on total muscle growth of the mice. However, it remains to be seen whether the effect of this bone defect on skeletal muscle is resolved with age. The mice used in the present study were between 2.5 and 3.5 months of age, and at this young adult stage it is conceivable that the initial differences in bone growth that resulted from the absence of MMP-9 may not yet have been resolved.

In summary, the architecture of skeletal muscle depends on the delicate balance of a number of regulatory processes. Here we have shown that growth and type of skeletal muscle fibers are altered by the absence of the inducible gelatinase MMP-9. The exact cause(s) of this difference is (are) not entirely clear, but may be a secondary consequence of defects in neural development and/or bone growth. Future studies will focus on how the absence of MMP-9 affects the ability of skeletal muscle to adapt to damage, hypertrophy, and other remodeling states in which MMP-9 expression and/or activity are known to increase dramatically.

\section{Acknowledgements}

This work was supported by a Young Investigator Award to Dr. David L. Allen from the American Physiological Society, a Research Endowment Award from the American College of Sports Medicine, and a KO1 Career Development Award (KO1 AR050505) from the National Institutes of Health.

\section{References}

Allen, D.L., A.S. Cleary, A.M. Hanson, S.F. Lindsay, J.M. Reed (2010a) CCAAT/enhancer binding factor-delta expression is increased in fast skeletal muscle by food deprivation and regulates myostatin transcription in vitro. Am J Physiol Regul Integr Comp Physiol, in press.

Allen, D.L., B.J. Greybeck, A.M. Hanson, A.S. Cleary, S.F. Lindsay (2010b) Skeletal muscle expression of bone morphogenetic protein-1 and tolloid-like-1 extracellular proteases in different fiber types and in response to unloading, food deprivation and differentiation. J Physiol Sci 60: 343-352.

Allen, D.L., B.C. Harrison, A. Maass, M.L. Bell, W.C. Byrnes, L.A. Leinwand (2001c) Cardiac and skeletal muscle adaptations to voluntary wheel running in the mouse. J Appl Physiol 90: 1900-1908.

Allen, D.L., G.E. McCall, A.S. Loh, M.C. Madden, R.S. Mehan (2010) Acute daily psychological stress causes increased atrophic gene expression and myostatin-dependent muscle atrophy. Am J Physiol Regul Integr Comp Physiol 299: R889-898.

Allen, D.L., D.H. Teitelbaum, K. Kurachi (2003) Growth factor stimulation of matrix metalloproteinase expression and myoblast $\mathrm{mi}$ gration and invasion in vitro. Am J Physiol Cell Physiol 284: C805-815.

-Armstrong, R.B., R.O. Phelps (1984) Muscle fiber type composition of the rat hindlimb. Am J Anat 171: 259-272.
Barnes, B.R., E.R. Szelenyi, G.L. Warren, M.L. Urso (2009) Alterations in mRNA and protein levels of metalloproteinases-2, -9 , and - 14 and tissue inhibitor of metalloproteinase- 2 responses to traumatic skeletal muscle injury. Am J Physiol Cell Physiol 297: C1501-C1508.

-Cannon, C.M., H. Dieter-Seelig, L.S. Stodieck, A.M. Hanson, S.J. Simske, V.L. Ferguson (2005) A novel combination of methods to assess sarcopenia and muscle performance in mice. Biomed Sci Instrum 41: 116-121.

Choi, Y. C., M. C. Dalakas (2000) Expression of matrix metalloproteinases in the muscle of patients with inflammatory myopathies. Neurology 54: 65-71.

Ducharme, A., S. Frantz, M. Aikawa, E. Rabkin, M. Lindsey, L.E. Rohde, F.J. Schoen, R.A. Kelly, Z. Werb, P. Libby, R.T. Lee (2000) Targeted deletion of matrix metalloproteinase- 9 attenuates left ventricular enlargement and collagen accumulation after experimental myocardial infarction. J Clin Invest 106: 55-62.

Fitts, R.H., K.S. McDonald, J.M. Schluter (1991) The determinants of skeletal muscle force and power: their adaptability with changes in activity pattern. J Biomech 24(suppl 1): 111-122.

Guérin, C.W., P.C. Holland (1995) Synthesis and secretion of matrix-degrading metalloproteases by human skeletal muscle satellite cells. Dev Dyn 202: 91-99.
Hanson, A.M., L.S. Stodieck, C.M. Cannon, S.J. Simske, V.L. Ferguson (2010) Seven days of muscle re-loading and voluntary wheel running following hindlimb suspension in mice restores running performance, muscle morphology and metrics of fatigue but not muscle strength. J Muscle Res Cell Motil 31: 141-153.

Harrison, B.C., D.L. Allen, B. Girten, L.S. Stodieck, P.J. Kostenuik, T.A. Bateman, S. Morony, D. Lacey, L.A. Leinwand, (2003) Skeletal muscle adaptations to microgravity exposure in the mouse. J Appl Physiol 95: 2462-2470.

Harrison, B.C., M.L. Bell, D.L. Allen, W.C. Byrnes, L.A. Leinwand (2002) Skeletal muscle adaptations in response to voluntary wheel running in myosin heavy chain null mice. J Appl Physiol 92: 313-322.

Ikonomidis, J.S., J.R. Barbour, Z. Amani, R.E. Stroud, A.R. Herron, D.M. Jr McClister, S.E. Camens, M.L. Lindsey, R. Mukherjee, F.G. Spinale (2005) Effects of deletion of the matrix metalloproteinase 9 gene on development of murine thoracic aortic aneurysms. Circulation 112(suppl 1I): I242-I248.

Ingber, D.E. (2006) Mechanical control of tissue morphogenesis during embryological development. Int J Dev Biol 50: 255-266.

Kaditis, A.G., E.I. Alexopoulos, A. Karathanasi, G. Ntamagka, S. Oikonomidi, T.S. Kiropoulos, E. Zintzaras, K. Gourgoulianis (2010) Adiposity and low-grade systemic inflammation modulate matrix metalloproteinase-9 levels in Greek children with sleep apnea. Pediatr Pulmonol 45: 693-699. 
Kherif, S., M. Dehaupas, C. Lafuma (1998) Matrix metalloproteinases MMP-2 and MMP-9 in denervated muscle and injured nerve. Neuropathol Appl Neurobiol 24: 309-319.

Kherif, S., C. Lafuma, M. Dehaupas, S. Lachkar (1999) Expression of matrix metalloproteinases 2 and 9 in regenerating skeletal muscle: a study in experimentally injured and $\mathrm{mdx}$ muscles. Dev Biol 205: 158-170.

Koskinen, S., M. Höyhtyä, T. Turpeenniemi-Hujanen, V. Martikkala, T. Makinen, J. Oksa, H. Rintamaki, M. Lofberg, H. Somer, T. Takala (2001a) Serum concentrations of collagen degrading enzymes and their inhibitors after downhill running. Scand J Med Sci Sports 11: 9-15.

Koskinen, S., W. Wang, A. Ahtikoski, M. Kjaer, X. Han, J. Komulainen, V. Kovanen, T. Takala (2001b) Acute exercise induced changes in rat skeletal muscle mRNAs and proteins regulating type IV collagen content. Am J Physiol Regul Integr Comp Physiol 280: 1292 1300.

Lewis, M.P., H.L. Tippett, A.C. Sinanan, M.J. Morgan, N.P. Hunt (2000) Gelatinase-B (matrix metalloproteinase-9; MMP-9) secretion is involved in the migratory phase of human and murine muscle cell cultures. J Muscle Res Cell Motil 21: 223-233.
Lluri, G., G.D. Langlois, P.D. Soloway, D.M. Jaworski (2008) Tissue inhibitor of metalloproteinase-2 (TIMP-2) regulates myogenesis and $\beta 1$ integrin expression in vitro. Exp Cell Res 314: 11-24.

Ovechkin, A.V., N. Tyagi, W.E. Rodriguez, M.R. Hayden, K.S. Moshal, S.C. Tyagi (2005) Role of matrix metalloproteinase-9 in endothelial apoptosis in chronic heart failure in mice. J Appl Physiol 99: 2398-2405.

Roy, R.R., K.M. Baldwin, V.R. Edgerton (1991) The plasticity of skeletal muscle: effects of neuromuscular activity. Exerc Sport Sci Rev 19: 269-312.

Rucavado, A., T. Escalante, C.F.P. Teixeira, C.M Fernandes, C. Dõaz, J.M. Gutierrez (2002) Increments in cytokines and matrix metalloproteinases in skeletal muscle after injection of tissue-damaging toxins from the venom of the snake Bothrops asper. Mediators Inflamm 11: 121-128.

Rullman, E., J. Norrbom, A. Stromberg (2009) Endurance exercise activates matrix metalloproteinases in human skeletal muscle. J App Physiol 106: 804-812.
Rullman, E., H. Rundqvist, D. Wågsäter, H. Fischer, P. Eriksson, C.J. Sundberg, E. Jansson, T. Gustafsson (2007) A single bout of exercise activates matrix metalloproteinase in human skeletal muscle. J Appl Physiol 102: 2346-2351.

Sternlicht, M. D., Z. Werb (2001) How matrix metalloproteinases regulate cell behavior. Annu Rev Cell Dev Biol 17: 463-516.

Tang, J., J. Liu, C. Zhou, J.S. Alexander, A. Nanda, D.N. Granger, J.H. Zhang (2004) MMP-9 deficiency enhances collagenase-induced intracerebral hemorrhage and brain injury in mutant mice. J Cereb Blood Flow Metab 24: 1133-1145.

Vu, T. H., J. M. Shipley, G. Bergers, J. E. Berger (1998) MMP-9/gelatinase B is a key regulator of growth plate angiogenesis and apoptosis of hypertrophic chondrocytes Cell 93: 411422.

Vu, T.H., Z. Werb (2000) Matrix metalloproteinases: effectors of development and normal physiology. Genes Dev 14: 2123-2133.

Zimowska, M., E. Brzoska, M. Swierczynska (2008) Distinct patterns of MMP-9 and MMP-2 activity in slow and fast twitch skeletal muscle regeneration in vivo. Int J Dev Biol 52: 307-314. 